\title{
VV Puppis in a low state: secondary-star irradiation or stellar activity?^
}

\author{
E. Mason ${ }^{1}$, S. B. Howell ${ }^{2}$, T. Barman ${ }^{3}$, P. Szkody ${ }^{4}$, and D. Wickramasinghe ${ }^{5}$ \\ 1 European Southern Observatory (ESO) Alonso de Cordova 3107, Vitacura, Santiago, Chile \\ e-mail: emason@eso.org \\ 2 NOAO, 950 N. Cherry Ave., Tucson, AZ, USA \\ e-mail: howell@noao.edu \\ 3 Lowell Observatory, Planetary Research Center, 1400 W. Mars Hill Rd., Flagstaff, AZ, USA \\ e-mail: barman@lowell .edu \\ 4 Dept. of Astronomy, University of Washington, Seattle, WA, USA \\ e-mail: szkody@astro.washington.edu \\ 5 Australian National University, Australia \\ e-mail: dayal@maths.anu.edu.au
}

Received 6 October 2007 / Accepted 6 August 2008

\begin{abstract}
Aims. Emission lines in polars show complex profiles with multiple components that are typically ascribed to the accretion stream, threading region, accretion spot, and the irradiated secondary-star. In low-state polars the fractional contribution by the accretion stream, and the accretion spot is greatly reduced offering an opportunity to study the effect of the secondary-star irradiation or stellar activity. We observed VV Pup during an exceptional low-state to study and constrain the properties of the line-forming regions and to search for evidence of chromospheric activity and/or irradiation.

Methods. We obtained phase-resolved optical spectra at the ESO VLT+FORS1 with the aim of analyzing the emission line profile and radial velocity as a function of the orbital period. We also tailored irradiated secondary-star models to compare the predicted and the observed emission lines and to establish the nature of the line-forming regions.

Results. Our observations and data analysis, when combined with models of the irradiated secondary-star, show that, while the weak low ionization metal lines ( $\mathrm{FeI}$ and $\mathrm{MgI}$ ) may be consistent with irradiation processes, the dominant Balmer $\mathrm{H}$ emission lines, as well as $\mathrm{NaI}$ and $\mathrm{HeI}$, cannot be reproduced by the irradiated secondary-star models. We favor the secondary-star chromospheric activity as the main forming region and cause of the observed $\mathrm{H}, \mathrm{NaI}$, and He emission lines, though a threading region very close to the $\mathrm{L} 1$ point cannot be excluded.
\end{abstract}

Key words. novae, cataclysmic variables - line: profiles - stars: chromospheres - stars: individiual: VV Puppis

\section{Introduction}

Different types of cataclysmic variables (CVs, i.e. dwarf novae, polars, etc.) in different states (outburst or quiescence) have shown Balmer emission lines forming close to the secondarystar. Depending on the system, plausible origins of these emission lines have been identified as the irradiated secondary hemisphere (e.g. Steeghs et al. 2001; Araujo-Betancor et al. 2003; Thoroughgood et al. 2005), or the accretion stream (e.g. Cowley et al. 1982; Mukai 1988; Schwope et al. 1997). More recently, it has been proposed that the Balmer emission lines in low-state polars could be the signature of chromosphere activity on the secondary-stars in AM Her, ST LMi, and EF Eri (Kafka et al. 2006, 2007; Howell et al. 2006b). This scenario is particularly intriguing if we consider that V471 Tau $\mathrm{H} \alpha$ emission line, previously thought to be caused by irradiation ${ }^{1}$ (Young et al. 1988), is best explained by stellar activity (Rottler et al. 2002).

In order to understand the source and formation mechanism of the observed Balmer emission lines in low-state polars, we

\footnotetext{
$\star$ Based on data collected on the ESO VLT within the program 272.D5044(A).

1 Young et al. (1988) use the word fluorescence in the broader context of conversion of high energy photons to low energy ones.
}

analyzed time-resolved optical spectra of VV Pup obtained during a low-state. The spectra were secured at the ESO VLT+FORS1 and have been already presented in Mason et al. (2007), where we focused on the white dwarf magnetic field signatures and, in particular, the first detection of the Zeeman absorptions. Here we present in Sect. 2 the data sample, in Sect. 3 our radial velocity study and the emission line profile analysis. In Sect. 4 the irradiated atmosphere model tailored for the VV Pup system is compared to the observations. Section 5 summarizes our results and conclusions.

\section{Data overview and major spectroscopic features}

The data were secured at the ESO VLT+FORS1 on 2004 March 16 and 22 (hereafter epoch 1 and epoch 2, respectively). The observation strategy and data reduction have already been presented in Mason et al. (2007), therefore we direct the reader to that paper for the details. Here we briefly present the two time series data sets and their observational characteristics. Each time series consists of 12 spectra and covers 1.26 orbital periods. The two series of spectra are very similar to each other as they both show strong cyclotron humps from the main and the secondary magnetic poles (see Fig. 1 in Mason et al. 2007) and similar 


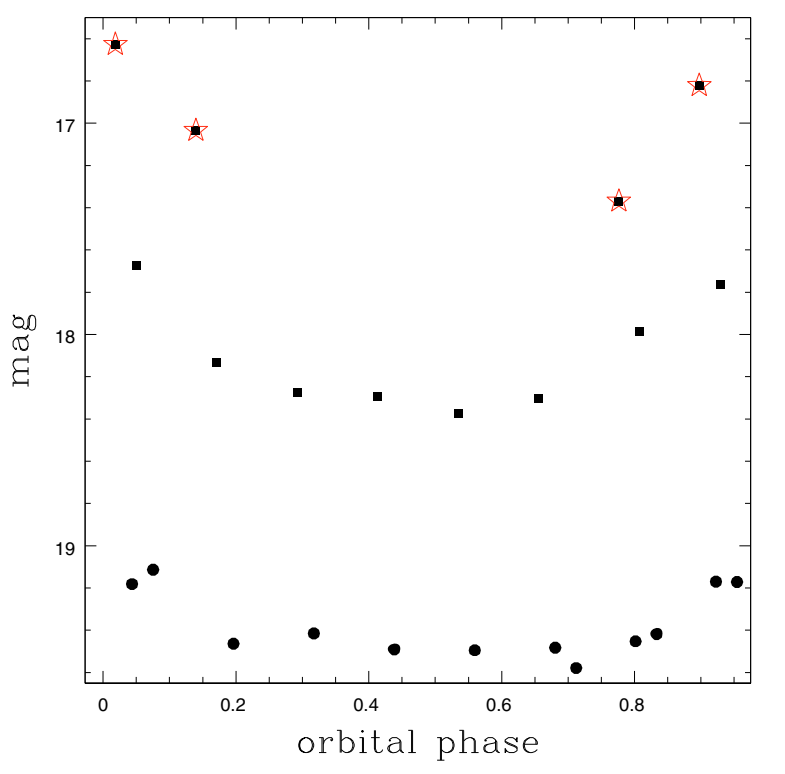

Fig. 1. Light curve extracted from the two data set by convolving each spectrum with a $V$-Johnson filter. Circles represents the data set of March 16. Squares represent the data set of March 22. Stars are for the last 4 frames in the March 22 data set which correspond to an increased mass transfer rate or to a flare episode.

emission lines (mainly HI, NaI and HeI, but also see below). The cyclotron hump emission modulates the system light curve (Fig. 1): VV Pup is brighter at the time of the secondary inferior conjunction when the main accreting pole on the white dwarf is in view, and fainter at the time of the secondary superior conjunction, when we face the white dwarf secondary magnetic pole.

However, the two time series data sets do have differences as they correspond to two different brightness states of the binary system. VV Pup was about 1 mag brighter during the epoch 2 observations than at the time of the epoch 1 observations. Moreover, epoch 2 observations showed a sudden increase in brightness in the last 4 spectra of our time series which corresponds to the phase range $0.80-1.15$. This is a possible signature of a flare and/or a sudden increase of the mass transfer rate (accretion burst), as we observe also an increase in flux of the emission lines (see Sect. 3).

Figure 2 shows the bright- and faint-phase spectrum in each series. Despite the different system brightness, corresponding spectra at the two epochs are similar. In particular, the brightphase spectra (orbital phase $\sim 0$ ) are dominated by cyclotron humps from the $32 \mathrm{MG}$ magnetic pole and just weak $\mathrm{H} \alpha$ and $\mathrm{H} \beta$ emission lines. The faint-phase spectra (orbital phase $\sim 0.5$ ) show cyclotron humps from the $55 \mathrm{MG}$ magnetic pole and relatively strong emission lines from $\mathrm{H}$, and low ionization energy metal lines. We recognize emission lines from the $\mathrm{MgI}(2)$ triplet (the strongest among the metal lines), the FeI (15) multiplet and the NaI D doublet. The latter is flanked, on the blue side, by the HeI (11) emission $\lambda 5876$ (Fig. 2's bottom panel).

\section{Emission line properties: observational evidence}

\subsection{Balmer emission lines}

In this section we analyze in detail the emission line properties (profile characteristics, radial velocities, and fluxes) to investigate their origin. We start with the Balmer emission lines which
Table 1. The best fit radial velocity curve solutions for the Balmer (narrow component) and the "non-Balmer" lines at the two epochs.

\begin{tabular}{ccccc}
\hline \hline Epoch & Line ID & R/B crossing & Systemic velocity & $K$ \\
\hline 1 & $\mathrm{H} \alpha$ & $0.489 \pm 0.001$ & $5 \pm 2$ & $277 \pm 2$ \\
1 & $\mathrm{H} \beta$ & $0.473 \pm 0.003$ & $-1 \pm 3$ & $267 \pm 3$ \\
2 & $\mathrm{H} \alpha$ & $0.497 \pm 0.001$ & $4 \pm 1$ & $267 \pm 1$ \\
2 & $\mathrm{H} \beta$ & $0.509 \pm 0.001$ & $-3 \pm 2$ & $283 \pm 2$ \\
& & & & \\
1 & FeI 5270 & $0.469 \pm 0.023$ & $-36 \pm 35$ & $326 \pm 19$ \\
1 & FeI 5328 & $0.493 \pm 0.028$ & $63 \pm 41$ & $313 \pm 22$ \\
1 & MgI 5170 & $0.447 \pm 0.007$ & $-43 \pm 11$ & $341 \pm 9$ \\
1 & NaI 5893 & $0.476 \pm 0.016$ & $2 \pm 21$ & $317 \pm 14$ \\
1 & HeI 5875 & $0.440 \pm 0.135$ & $-38 \pm 190$ & $250 \pm 66$ \\
& & & & \\
2 & FeI 5270 & $0.498 \pm 0.020$ & $7 \pm 40$ & $371 \pm 38$ \\
2 & FeI 5328 & $0.471 \pm 0.082$ & $-1 \pm 132$ & $289 \pm 46$ \\
2 & MgI 5170 & $0.478 \pm 0.010$ & $-12 \pm 12$ & $329 \pm 11$ \\
2 & NaI 5193 & $0.468 \pm 0.015$ & $-11 \pm 20$ & $323 \pm 16$ \\
2 & HeI 5875 & $0.568 \pm 0.008$ & $6 \pm 6$ & $178 \pm 5$ \\
\hline
\end{tabular}

$\dagger$ Barycenter of the blend $\operatorname{MgI}(2) \lambda \lambda 5167,5173$.

$\dagger$ Barycenter of the blend NaI D $\lambda \lambda 5190,5196$.

have higher $\mathrm{S} / \mathrm{N}$ and, therefore, provide more robust measurements.

Figure 3 shows the profile of both the $\mathrm{H} \alpha$ and $\mathrm{H} \beta$ emission lines at the two epochs. The line intensity varies with the orbital phase being stronger around phase 0.5 (see also Fig. 5), while the line profile depends on the epoch. The emission lines appear as mostly single Gaussian (or single component) emission lines in the epoch 1 spectra, while clearly multiple components ( 2 or 3 ) are present in epoch 2 spectra. In order to assess the significance of multiple components at both epochs we fit each line of each spectrum both with a single and multiple (2 to 3 ) Gaussians. We then compared the significance of the multiple fit with respect to the single Gaussian fit with the F-test. We eventually adopted a multiple Gaussian fit whenever its probability exceeded $\geq 3.5 \sigma$. The result is that in epoch 1 the $\mathrm{H} \alpha$ emission lines are better fit by a double Gaussian in $75 \%$ of the cases (i.e. 9/12 spectra), while the $\mathrm{H} \beta$ line is better fit by a single Gaussian in all but one case (where there was no detection of the $\mathrm{H} \beta$ emission line, 11/12 spectra or $92 \%$ ). In epoch 2 a multiple Gaussian fit ( 2 or 3 ) is needed in $93 \%$ of the cases (10/12 spectra) for the $\mathrm{H} \alpha$ emission line and in $25 \%$ of the cases (3/12 spectra) for the $\mathrm{H} \beta$ line. The radial velocities corresponding to each component are shown in Fig. 4 where solid circles represent the main narrow component (FWHMs are of the order of 2-3 $\AA$ ), while the open circles are for the secondary, weaker component ${ }^{2}$. Similar weaker components have also been identified in AM Her (Kafka et al. 2006), EF Eri (Howell et al. 2006b) and ST LMi (Kafka et al. 2007) and called "satellite" lines. Figure 4 shows that the main narrow component has a clean sinusoidal pattern and is in phase with the secondary-star. We fitted the $\mathrm{H} \alpha$ and the $\mathrm{H} \beta$ main narrow components at each epoch. The best fit parameters are shown in Fig. 4 as well as in Table 1.

The best fit parameters in Table 1 appear quite similar to each other. From here on, however, we regard the epoch $1 \mathrm{H} \beta$ radial velocity solution as the most reliable for showing the line location within the binary system as it is not biased at any phase by a secondary emission component. Because of the phasing of the

\footnotetext{
2 Note that the FWHM of the secondary component in the epoch 1 spectra is not larger than $2 \AA$, while, in epoch 2 spectra it varies (with the orbital phase) between 2 and $6 \AA$.
} 


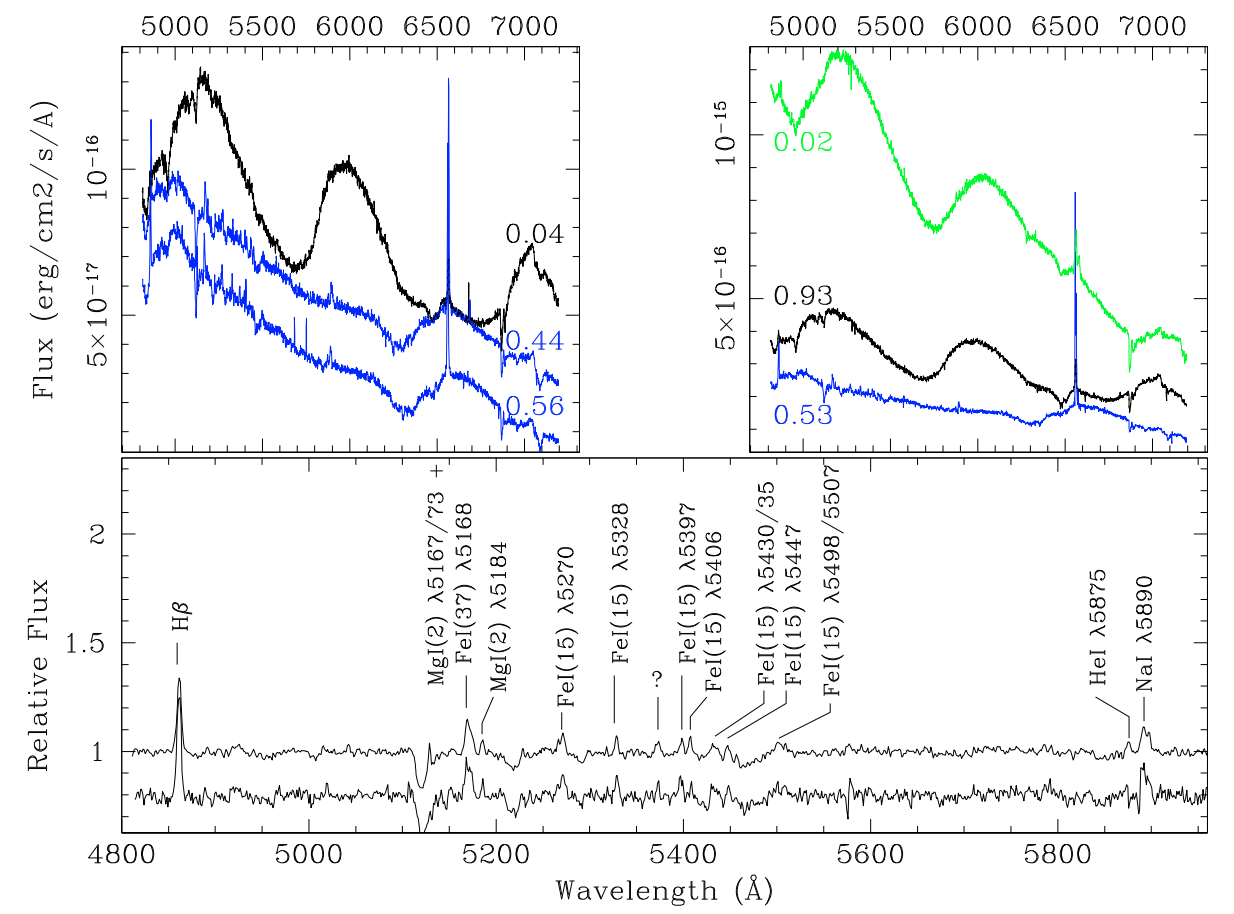

Fig. 2. Top panels: bright- (black) and faint- (blue) phase spectra of each data set, epoch 1 on the left and epoch 2 on the right. The numbers near each spectrum indicate their orbital phase. In the top left panel the spectrum at orbital phase 0.56 has been shifted vertically by the constant $-0.2 \mathrm{e}-$ $16 \mathrm{erg} \mathrm{cm}^{2} \mathrm{~s}^{-1}$. In the top right panel the green spectrum is the bright-phase spectrum observed during the accretion burst. Bottom panel: a zoomed-in view of the 4800-5960 $\AA$ region with the identification of the low ionization energy metal lines. The spectra in the bottom panel have been normalized. The top spectrum (in the bottom panels) is the average of the spectra at orbital phases 0.44 and 0.56 (properly shifted before co-addition) of epoch 1 data set; while the bottom spectrum is that at orbital phase 0.53 in epoch 2 data set. The bottom spectrum in the bottom panel has been vertically shifted by the constat -0.25 .
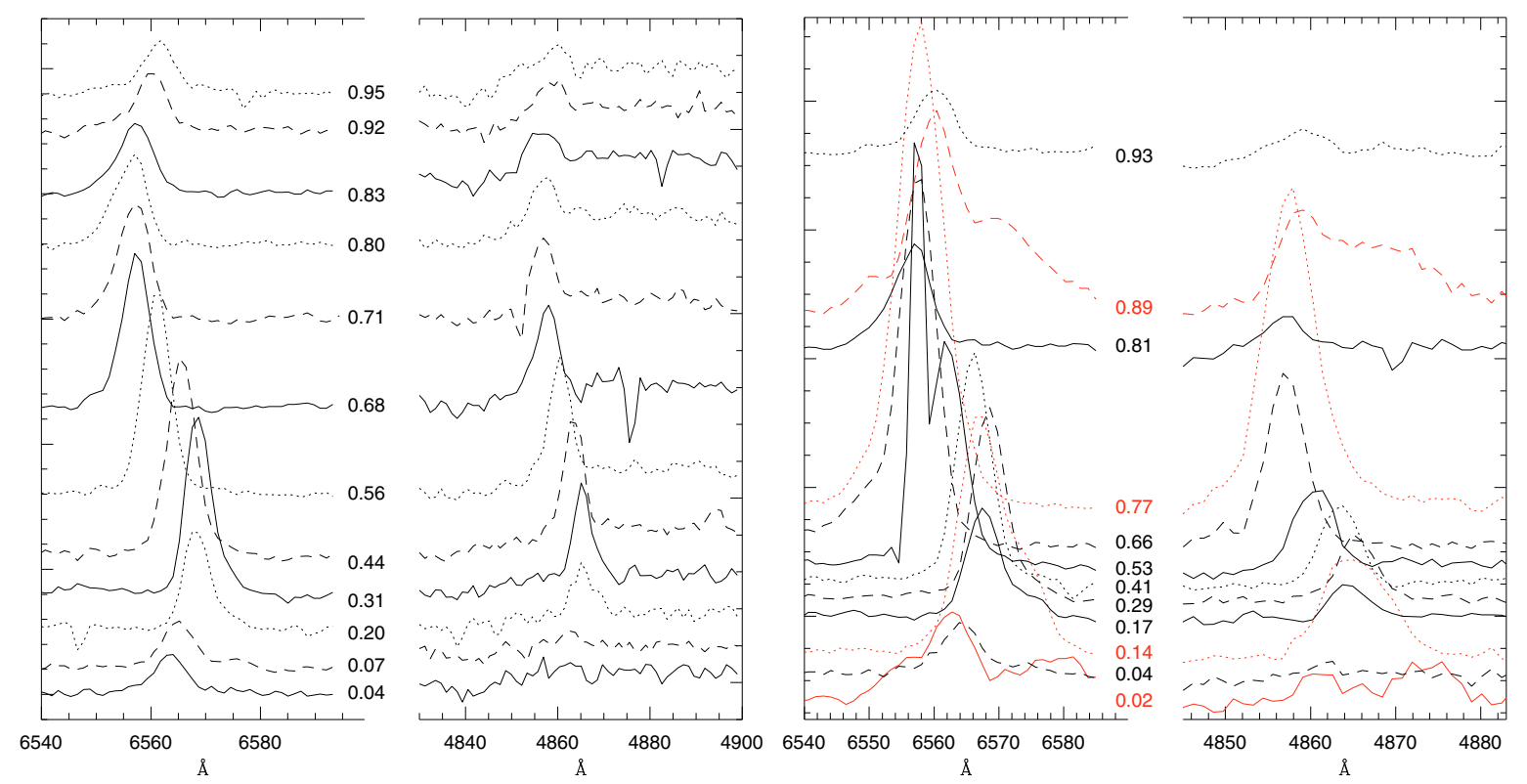

Fig. 3. Balmer emission line profiles for epoch 1 (left panel) and epoch 2 (right panel). Different line styles are just for clarity, while the lines in red color correspond to the four epoch 2 flaring/bursting spectra.

radial velocity curve, it is tempting to associate the line with the secondary-star and conclude that it mirrors the secondary-star orbital motion. However, Howell et al. (2006a) have determined the secondary-star radial velocity curve by measuring the $\mathrm{NaI}$ absorption at 2.067 micron. A comparison of our primary, narrow emission line radial velocity fit with that by Howell et al. (2006a), shows that we agree in the phase of the red to blue crossing (i.e. the secondary superior conjunction), but not in $K$ amplitude, nor in the $\gamma$ velocity determinations. Our Keplerian velocity is significantly smaller than that given by Howell et al. (2006a) while our $\gamma$ velocity is $\sim 140 \mathrm{~km} \mathrm{~s}^{-1}$ more positive. We can attempt to reconcile the measurements by applying the inverse $K$ velocity correction to our determination on the hypothesis that the Balmer emission lines originate from the heated face of the secondary-star. Assuming the most extreme unrealistic case of the emission line forming just at the L1 point (Horne \& Schneider 1989) we derive $K \sim 408 \mathrm{~km} \mathrm{~s}^{-1}$, which is only $6 \mathrm{~km} \mathrm{~s}^{-1}$ smaller than the Howell et al. (2006a) result. The more realistic assumption of the emission line rising from about half the secondary-star surface produces $K=323 \mathrm{~km} \mathrm{~s}^{-1}$ which is significantly smaller (by $\sim 90 \mathrm{~km} \mathrm{~s}^{-1}$ ) than Howell et al. (2006a) result. Moreover, this scenario still leaves unexplained the significant difference in the $\gamma$ velocities.

The study carried out by Mukai (1988) has shown that in the case of (high rate) mass transfer we can expect emission lines phased with the secondary-star either from the horizontal part of the accretion stream (before the coupling region where the gas gets funneled by the white dwarf magnetic field lines), or in the rising part of the funneled gas just after (or at) the threading region (emission lines from the gas falling onto the white dwarf 


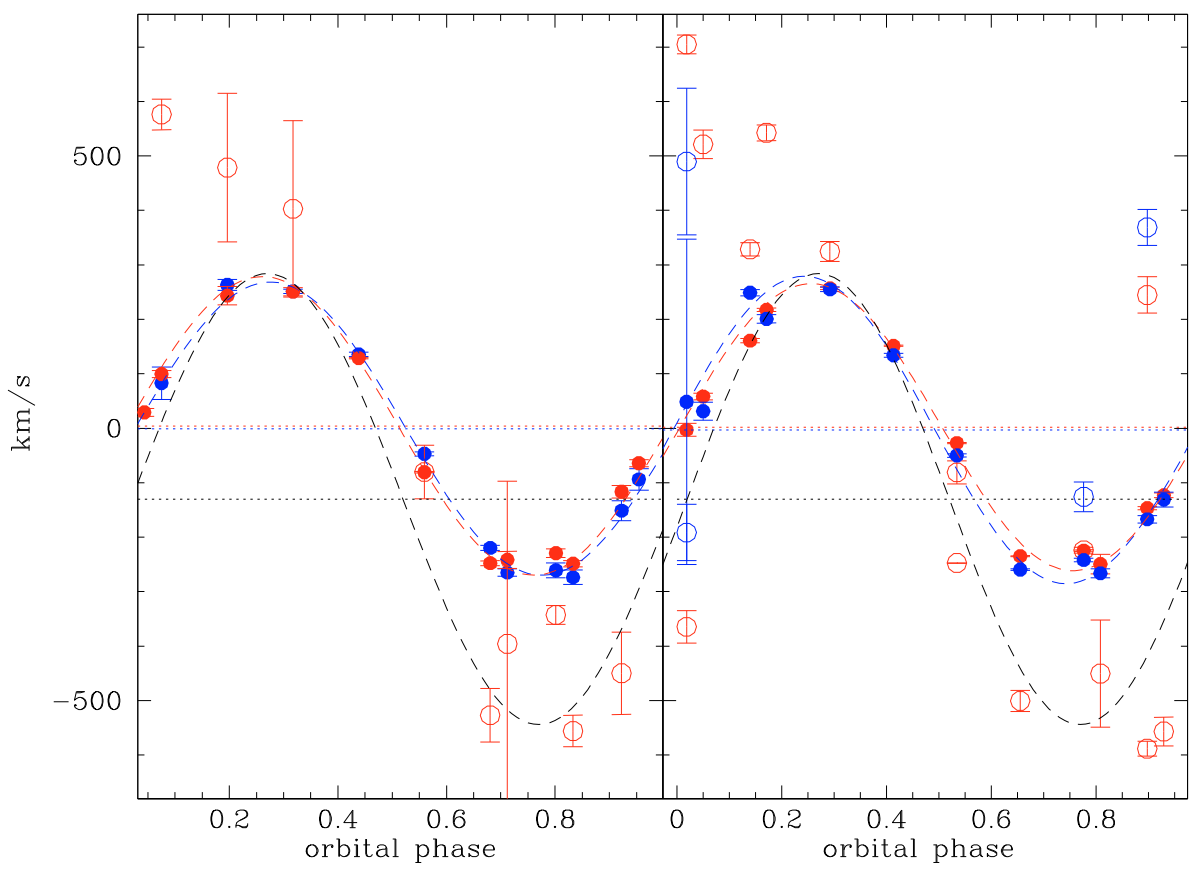

Fig. 4. Radial velocity measurements (left epoch 1, right epoch 2) of the Balmer lines $\mathrm{H} \alpha$ (red solid circles) and $\mathrm{H} \beta$ (blue solid circles) and their best fit (red and blue dashed lines). Open circles represent the secondary-satellite component (same color convention), while the dotted horizontal lines represent the $\gamma$ velocity as determined by the sinusoidal fit. In black we report the best fit radial velocity curve determined by Howell et al. (2006a, sinusoidal dashed line) and its $\gamma$ velocity (horizontal dotted line). along the magnetic field lines will necessarily be phased with the primary-star).

In the case of emission lines from the horizontal stream, we should observe $K$ amplitudes which are larger than $K 2$, the real secondary-star Keplerian velocity. The red-to-blue crossing phase and $\gamma$ velocity should be similar to those of the real secondary-star radial velocity curve. Hence we can exclude emission from the horizontal stream before it is funneled in the magnetic field lines as the cause of the narrow main $\mathrm{H}$ emission line source.

In the specific case of the VV Pup low-state, it is difficult to imagine a line-forming region just on the rising part of the gas that has been funneled along the magnetic field lines, as one would also expect that emission lines would form in the falling part and the horizontal stream (if present), as well as in the impact region. We do not observe either one. We could expect emission lines from the threading region provided that this happens to be very close to the secondary-star. This might actually be the case for short orbital period systems and low-state systems (the magnetosphere radius is inversely proportional to the mass transfer rate to the power of $-2 / 11$, hence it moves outward and far from the primary star during low-states. See Warner 1995 for a review and Mukai 1988 for details.). However, the high velocities $\left(\sim 300 \mathrm{~km} \mathrm{~s}^{-1}\right)$ and the chaotic motion of the gas in the threading region are quite inconsistent with our observed FWHMs and possibly also (but not necessarily) with our derived $\gamma$ velocity.

Hence, we favor the hypothesis of an extended atmosphere and/or chromospheric prominence-loops from the secondarystar. Prominence-loops can easily be out of the binary orbital plane thus explaining (in analogy with Mukai consideration of the funneled accretion stream) both the small $K$ amplitude, the relatively small line width and the (more positive) $\gamma$ velocity. In the hypothesis that these prominence-loops are somehow triggered or enhanced by the white dwarf magnetic field (Howell et al. 2006b; see also Uchida \& Sakurai 1985, Fig. 1) it is reasonable that they would not be visible during the secondary-star inferior conjunction. With regard to this, we show in Fig. 5 the modulation of the emission line flux versus the orbital period. We identify a well defined bell shape distribution for epoch 1 measurements (left panel, red and blue symbols for $\mathrm{H} \alpha$ and $\mathrm{H} \beta$, respectively) as expected for lines which form on just one side of a rotating star. The more scattered distribution for epoch 2 measurements (Fig. 5 right panel, red and blue symbols for $\mathrm{H} \alpha$ and $\mathrm{H} \beta$, respectively) is explained both by the bias introduced by the 4 flaring/bursting spectra (starred symbols) as well as by the overall line profile which shows a more extendend (/multiple) line-forming region(s) than in epoch 1 spectra; i.e. multiple components.

\subsection{The non-Balmer emission lines}

We can attempt a similar analysis for the weaker non-Balmer emission lines, namely the metal emission lines (MgI and FeI), $\mathrm{NaI}$ and HeI. As they are weak and often blended (e.g. the MgI 5167, 5173 and, possibly, FeI 5168 are all blended together), and given the instrumental resolution, we limit our measurements to the line flux barycenter and the integrated flux. We also limit our measurements to the two FeI lines $\lambda 5270$ and $\lambda 5328$, which appear to be the strongest among multiplet (15).

We plot in both Figs. 5 and 6 the flux measurements of the HeI and the low ionization energy emission lines. Figure 5 shows the flux modulation of the weak lines in comparison to the Balmer lines, while Fig. 6 shows only the weak, non-Balmer lines in a rescaled plot for clarity. Note that the FeI measures were not plotted in Fig. 5 (again for clarity).

The figures show that the low ionization energy metal lines and the $\mathrm{NaI}$ and the HeI emission lines in epoch 1 mirror the Balmer line behavior and follow a bell shaped modulation throughout the orbital period. In epoch 2 , however, though we are still seeing the emission lines generally strongest around secondary superior conjunction, the flux distribution is more scattered. The measured line flux is at least double with respect to epoch 1 for all the lines, implying that they are all affected by the brighter state recorded in epoch 2 . We note that the HeI emission line is different from the other lines in that it also becomes strong and visible in the bursting spectra at orbital phase $0.8-1.1$, similar to the Balmer lines (Fig. 5).

The radial velocities for each of the measured lines and their best fits are displayed in Fig. 7 and Table 1, respectively. These 

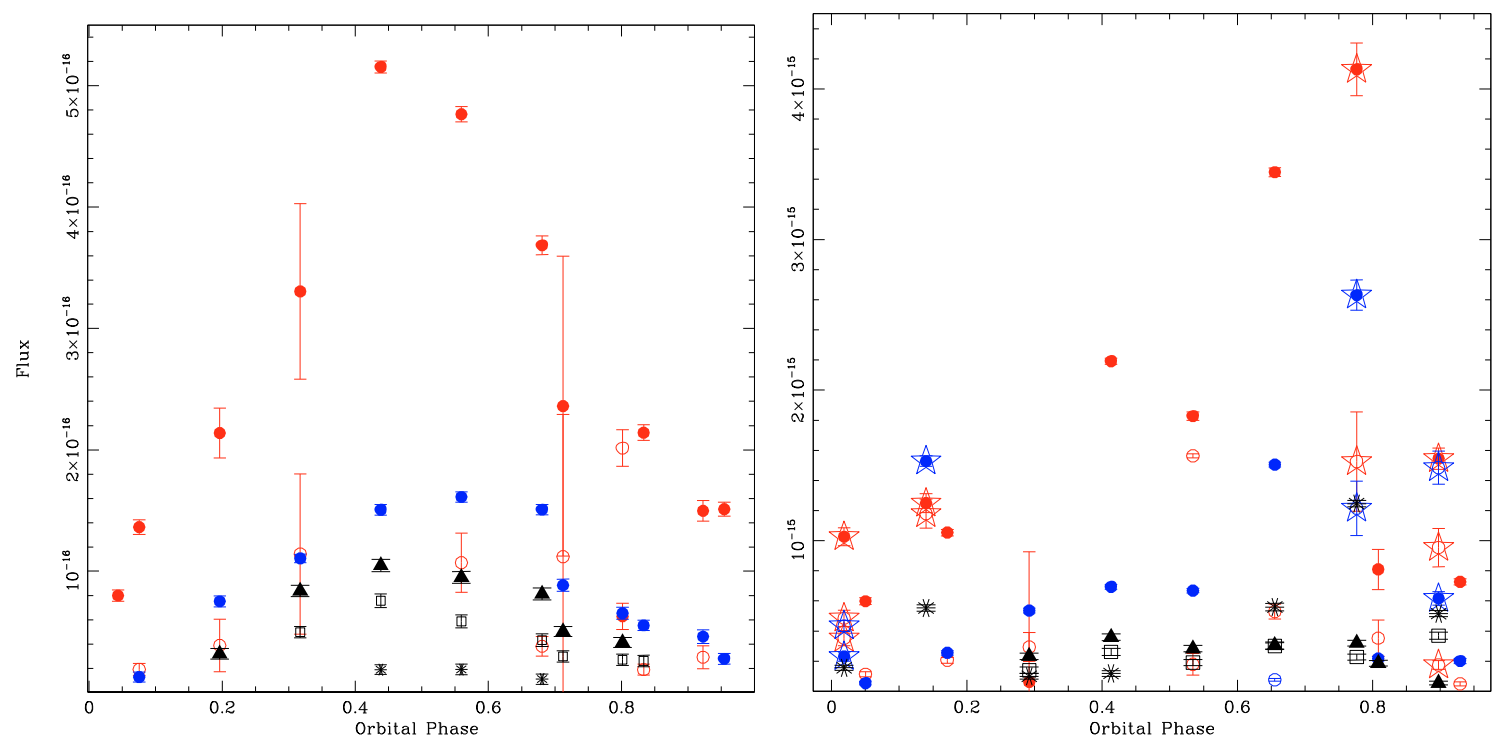

Fig. 5. Emission line flux modulation versus the orbital period. Epoch 1 data are in the left panel, while epoch 2 data are displayed in the right panel. In both panels, red solid circles are for the $\mathrm{H} \alpha$ main-narrow component, while red outlined circles are for any of the additional components found via Gaussian fit. Similarly, blue solid and empty circles refer to the $\mathrm{H} \beta$ emission lines. Black triangles are for the $\mathrm{MgI}$ line, empty black squares for the NaI doublet, and black asterisks for the HeI line. In the right panel, the starred symbols mark the Balmer emission lines in the 4 flaring/bursting spectra of epoch 2.
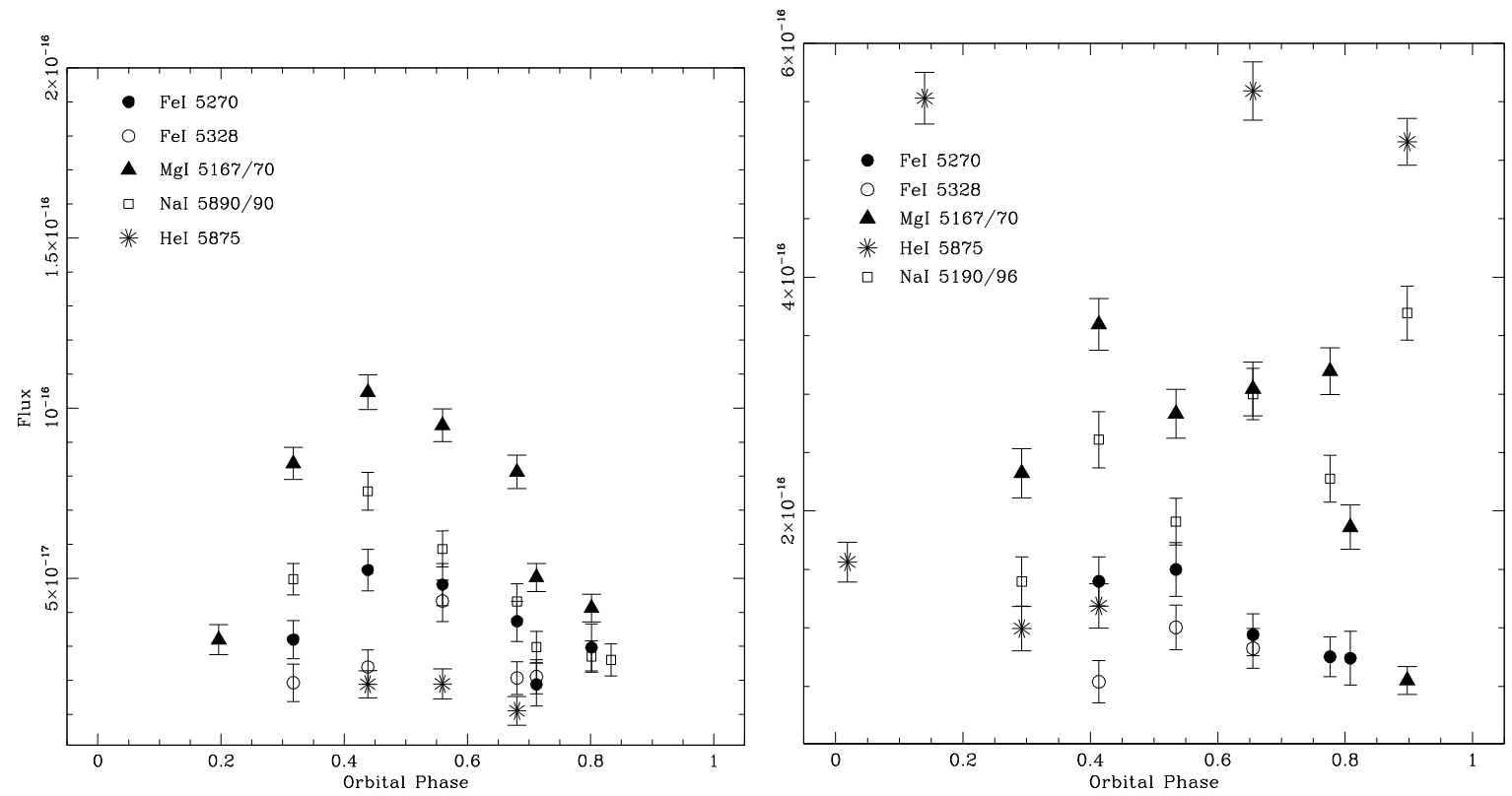

Fig. 6. Zoomed-in view of the emission line flux distribution versus orbital phase. The left and the right panels display the data of epoch 1 and 2 , respectively. Different symbols are for different emission lines (notation on the figure itself). Note that in the case of the HeI emission the data point at orbital phase 0.78 has not been plotted for clarity, being a factor of two brighter than the brightest points displayed.

are characterized by larger errors. However, the overall velocity curves are very similar to those derived for the Balmer lines with the only difference being a possibly larger amplitude. We can imagine a line-forming region closer to the secondary-star and the L1 point in the case of the non-Balmer emission lines, or a temperature gradient in the line-forming region. We note that the HeI emission lines in epoch 2, again shows a very different behavior with respect to the other lines and epoch 1 measurements. A fit of the HeI lines shows a much smaller amplitude implying that the HeI lines, in the bursting spectra, form closer to the white dwarf but still on the secondary-star side of the binary system center of mass. The phase of the red to blue crossing is delayed as observed in the radial velocity measurements during high states (e.g. Cowley et al. 1982; Diaz \& Steiner 1994; Schneider \& Young 1980). The HeI lines appear significantly stronger in the bursting spectra and this seems consistent with a picture where the emitting/threading region gets hotter due to a higher mass transfer rate and denser gas.

\section{The irradiated secondary-star model}

Above we have shown that the low-state emission lines observed in VV Pup are consistent with an origin on or near the secondarystar. Three scenarios for their production have been postulated in 

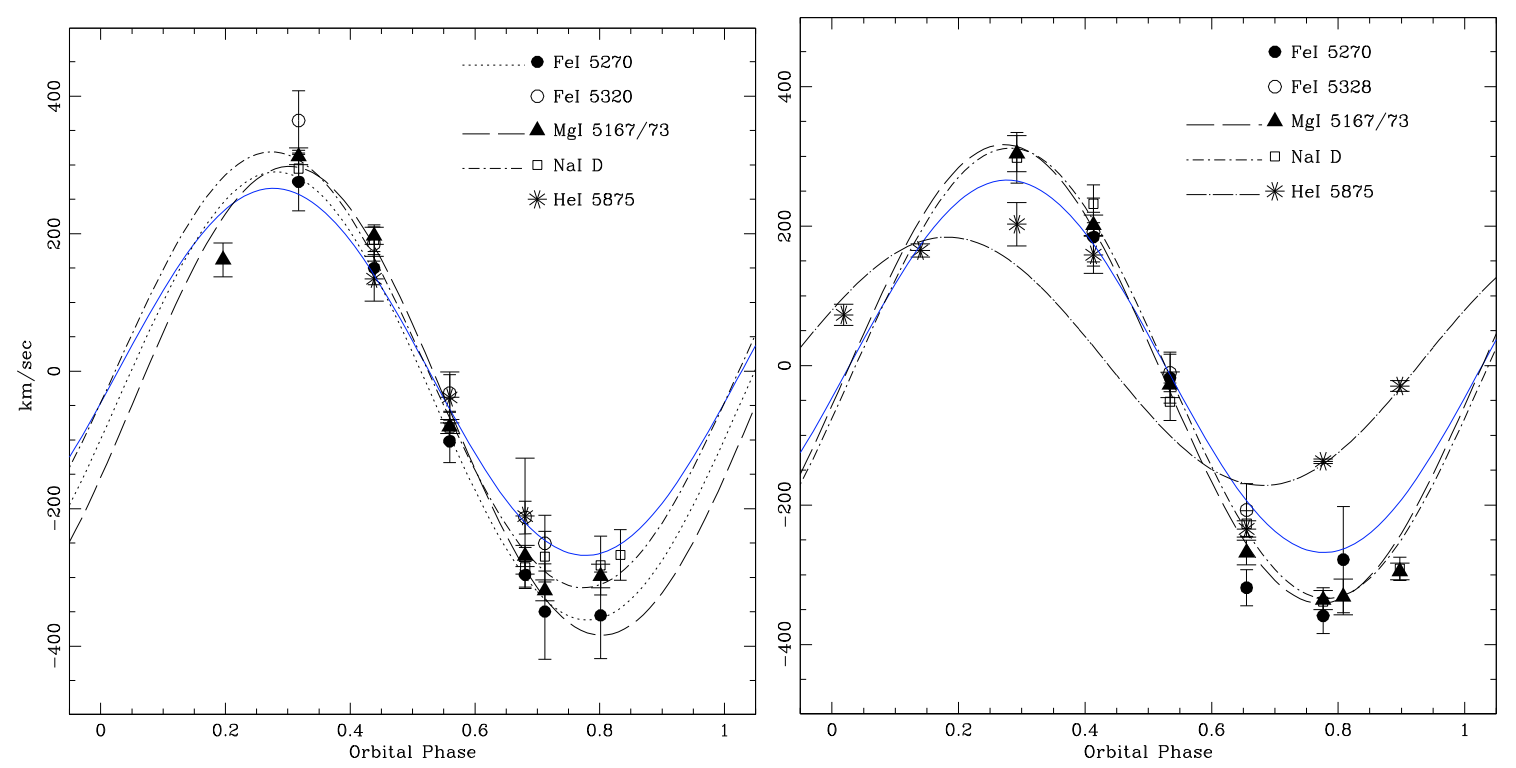

Fig. 7. Radial velocity measurements of the low ionization metal lines, ( $\mathrm{MgI}$ and $\mathrm{FeI}$ ) and the $\mathrm{NaI}$ and $\mathrm{HeI}$ emission lines. Left panel is for epoch 1 while the right panel is for epoch 2. Different symbols are for different emission lines and the notation is on the figure itself. Different line styles represent the best fit (as in Table 1) for the specified line. Note that not all best-fit radial velocity curves have been plotted for clarity. In both panels the blue solid line is the best fit determined for the $\mathrm{H} \beta$ emission in epoch 1 .

the literature: threading region/accretion stream components, irradiation, and stellar activity. Though some of our observational evidence may favor the threading region and/or the stellar activity scenario (see Sect. 3), we could not completely rule out the irradiation case. We now want to test the irradiation hypothesis by producing synthetic models of the secondary-star irradiated by the white dwarf.

A number of papers, both about close binaries and CVs, talk about irradiation and present spectra containing narrow emission features attributed to that (see Barman et al. 2004, and reference therein). Calculations of the energy needed for irradiation as well as that supplied by the white dwarf have been presented as well (e.g., Rottler et al. 2002; Schmidt et al. 1995). Models of irradiated spectra have reached a sophisticated level in Barman et al. (2004), where it was shown that, in the case of pre-CV binaries having white dwarfs of $T_{\mathrm{eff}}>20000 \mathrm{~K}$, the atmosphere of the secondary facing the white dwarf contains a temperature inversion that leads to strong emission lines and shallow absorption features. For lower temperature white dwarfs $\left(T_{\text {eff }}<20000 \mathrm{~K}\right)$, it was shown that the temperature inversion in the secondary-star's upper atmosphere produces only weak, narrow emission lines from low excitation ionized metals (mostly FeI). Furthermore, since the temperature inversion in cooler systems is confined to a part of the atmosphere well above the photosphere, photospheric absorption lines and bands are unaffected.

Following the model procedure outlined in Barman et al. (2004), the white dwarf in VV Pup was modeled with a temperature of $11900 \mathrm{~K}$ and $\log g=8.0 \pm 0.5$ (Araujo-Betancor et al. 2005). The secondary-star was modeled assuming it to be a normal, solar metallicity main sequence star of spectral type M7 (Howell et al. 2006a), $T_{\text {eff }}=3000 \mathrm{~K}$ and $\log g=5.25$. The secondary-star temperature and gravity were determined by fitting the $2.067 \mu \mathrm{m} \mathrm{NaI}$ absorption line profiles presented in Howell et al. (2006a). The Na I line and its local continuum were assumed to be unaffected by the white dwarf flux as the $\sim 12000 \mathrm{~K}$ white dwarf contributes less than $1 \%$ of the $K$-band flux in VV Pup. Additionally, even if irradiation is present, the
$2.067 \mu \mathrm{m} \mathrm{Na}$ I absorption line will be unaffected as it forms deep in the M star photosphere, below the atmospheric layer at which any temperature inversion would occur. Using these values and the additional system parameters presented in Howell et al. (2006a), we produced VV Pup model spectra (without cyclotron humps) by summing white dwarf template spectra of different temperatures with the irradiated M7V LTE atmosphere model. Figure 8 shows two of these models (black dashed lines): one having a $20000 \mathrm{~K}$ white dwarf and the other having a $11900 \mathrm{~K}$ white dwarf. In both cases the white dwarf and the irradiated secondary spectra have been rotationally broadened to 25 and $200 \mathrm{~km} \mathrm{~s}^{-1}$, respectively, as appropriate for VV Pup. They also assume orbital phase 0.5 (a phase where the irradiation is expected to be the largest) and orbital inclination $i=90^{\circ}$. Since VV Pup has an orbital inclination $i \sim 75^{\circ}$, the models line strength are an upper limit for the expected irradiation.

Our secondary-star model assumes a spherical star, i.e. the Roche geometry is not accounted for. Because of this, a small region of the L1 area of the secondary-star atmosphere will be slightly closer $\left(\sim 0.1 \mathrm{R}_{2}\right.$, see Fig. 6 in Howell et al. 2000$)$ to the primary and have lower gravity, than in our spherical photosphere model. In order to explore the consequences of neglecting the exact Roche geometry, we computed several additional models artificially moving the secondary-star closer (but no closer than L1) to the $11900 \mathrm{~K}$ white dwarf and arbitrarily lowering its surface gravity. Each of these tests produced only slightly greater temperature inversions, without altering the overall irradiated spectrum appearance and our conclusions.

Also, since the NLTE assumption is more appropriate in the case of an external radiation source, we also produced a model having a $11900 \mathrm{~K}$ white dwarf and a NLTE irradiated secondary atmosphere (black solid line in Fig. 8). This latter model is the one directly compared to our VV Pup observations (blue solid line in Figs. 8 and 9). We might note that the use of LTE secondary-star atmosphere models in place of the more proper NLTE one, does not alter the conclusion as they predict the same "forest" of low excitation energy metal lines, though with reduced strengths. Figure 9 shows a zoomed-in view of the 

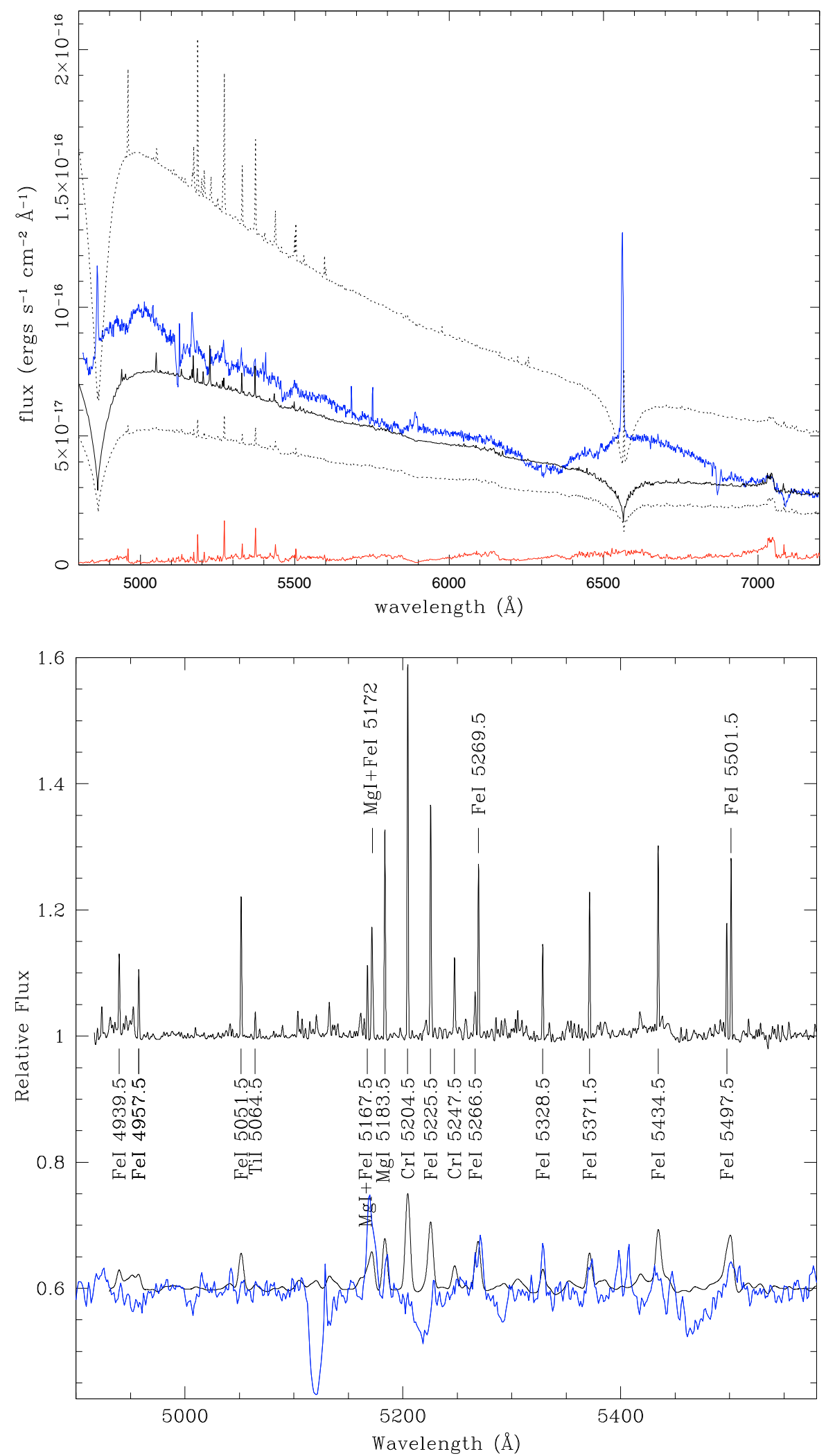

Fig. 8. Models vs. observation comparison. The black spectra are the three irradiation models (white dwarf + irradiated secondary star) described in Sect. 4. The dashed lines are for the LTE secondary atmosphere models, while the solid line is for the NLTE secondary atmosphere model. The top spectrum is for a $20000 \mathrm{~K}$ white dwarf, while the two bottom ones are for a $11900 \mathrm{~K}$ white dwarf. In blue color is the VV Pup epoch 1 faint phase spectrum. It is the average of the two spectra at phase 0.44 and 0.56 (see Fig. 2). We also show, in red color, the NLTE irradiated secondary atmosphere model alone. Flux units refer to the observed spectrum. The four models have been arbitrarily scaled. However, the NLTE irradiated secondary-star model (red) and the NLTE irradiated secondary-star model + the $11900 \mathrm{~K}$ white dwarf (solid black) have been scaled by the same factor so that their relative flux and fractional contribution is preserved.
Fig. 9. Comparison of model and observation in the 4900-5600 $\AA$ region. In black color are the normalized model spectra at spectral resolution $0.5 \AA$ (the resolution in the stellar atmosphere code, top) and $5.0 \AA$ (about the resolution of our optical spectra, bottom). In blue color we plot the normalized faint phase spectrum of epoch 1 , corresponding to the average of the two faint phase spectra at orbital phase 0.44 and 0.56 . All the spectra have been normalized and shifted to a common reference wavelength. The observed blue spectrum and the low resolution model have been vertically shifted by the constant -0.4 for clarity.
4900-5600 ^ region comparing the average normalized faintphase epoch 1 spectrum (already shown in Fig. 2) with the NLTE model, both at spectral resolution $0.5 \AA$ and resolution matching our observations. Figure 9 shows that there is not a one-to-one matching of the observed and predicted lines and, in particular, that we do not observe CrI and TiI emissions. This might at least be explained in part by the model assumptions (see also below), as well as by the Zeeman absorption and the cyclotron humps, which "bias" our observed spectra.

Qualitatively we note that the irradiation models predict metal emission lines mainly from FeI, TiI, MgI and CrI (i.e. low excitation, singly ionized metals). These metal lines are predicted to get stronger for higher white dwarf temperatures as the increase in UV flux can push the temperature inversion 
deeper into the cool star's atmosphere. Balmer, HeI, and NaI emission lines due to irradiation are not expected in the case of a $\sim 12000 \mathrm{~K}$ white dwarf as in VV Pup (see Fig. 8), while only $\mathrm{H}$ and $\mathrm{NaI}$ emissions are expected for white dwarf temperatures $\geq 20000 \mathrm{~K}$.

Recent UV observations have pointed out the presence of a "hot spot" on the white dwarf surface (Szkody et al. 2006; Schwope et al. 2007) for low-state polars. This hot spot seems to have a temperature as high as $18000-24000 \mathrm{~K}$ and is present in all the low-state magnetic systems observed in the UV up to now (e.g. AM Her Gänsicke et al. 2006). Araujo-Betancor et al. (2005) determined a slightly better fit to their UV spectra by including a second hotter component superimposed on the white dwarf model spectrum (either a power law or a second white dwarf spectrum), though they could not constrain its size and temperature. We have verified that model calculations which include a $25000 \mathrm{~K}$ hot spot covering about $1 \%$ of the white dwarf surface and superposed on the VV Pup $11900 \mathrm{~K}$ white dwarf, can indeed produce enhanced $\mathrm{H}$ emission together with a low ionization metal-line forest. A $25000 \mathrm{~K}$ hot spot model does not noticeably affect the white dwarf optical continuum but produces too weak $\mathrm{H}$ emission lines to match our observations. In addition, the model predicts an inverse Balmer decrement, which is also in disagreement with our observations. We note that the relative strengths of the produced emission lines depend on several factors such as the NLTE-effects (which alters the Boltzmann distribution of the population levels), the assumed abundances (in our case solar abundances), and atomic parameters (e.g. oscillator strengths and/or line centers). Still, within reasonable assumptions, these models do not seem capable of fully explaining the observations. At the same time, our data cannot independently provide evidence for or against the presence of a hot spot on the white dwarf. Only low-state UV observations similar to those of, e.g., EF Eri (Szkody et al. 2006; Schwope et al. 2007) can solve this issue. We would like to point out that the EF Eri optical spectrum is well fit by just a $\sim 10000 \mathrm{~K}$ white dwarf model, which fails to fit the UV data (Schwope et al. 2007).

\section{Summary and conclusions}

Phase-resolved optical spectroscopy of the polar VV Pup during a low-state has shown the presence of Balmer $\mathrm{H}$, FeI, MgI, NaI and HeI emission lines, which form on the side of the secondary-star facing the white dwarf. Their radial velocity curves are roughly consistent with each other but different from the secondary-star radial velocity curve measured by Howell et al. (2006a). Also, the application of the inverse $K$-correction is insufficient to produce agreement between our and the Howell et al. radial velocity.

The emission line fluxes show a bell shaped modulation across the orbit during epoch 1 observations, which is often taken as irradiation of the secondary-star. We produced irradiated secondary-star models tailored for VV Pup and, though irradiation predicts low ionization metal lines, it does not explain the Balmer, $\mathrm{Na}$ and HeI emission lines strengths we observed. In particular, to produce these emission lines would require a $>20000 \mathrm{~K}$ white dwarf in VV Pup, contrary to the UV observations. The possible presence of a hot spot $(20000$ to $24000 \mathrm{~K})$ on the magnetic white dwarf, similar to that observed in low-state polars such as EF Eri and AM Her, could alternatively provide some Balmer, $\mathrm{Na}$ and possibly He emission lines. However, in epoch 2 observations taken while VV Pup was experiencing an increase in mass transfer, we observed the same lines to strengthen and show a more scattered flux distribution with the orbital period. This also disfavors irradiation of the secondarystar.

Our observations are better explained by a scenario in which the $\mathrm{H}, \mathrm{Na}$ and $\mathrm{HeI}$ emission lines form mainly on the white dwarf facing side of the secondary-star either in the threading region close to the L1 point, or in chromospheric prominenceloops possibly shocked near the location of the white dwarf magnetospheric radius. We note that the NaI doublet and the $\mathrm{H} \alpha$ emission lines are known to be good indicators of chromosphere activity in M and L dwarfs (Andretta et al. 1997; Schmidt et al. 2007). Flux ratios of the HeI $\lambda 5875 / \mathrm{HeI} \lambda 6678 \sim 2.5-3.5$, as measured by us in the "bursting" spectra of epoch 2, are consistent with low density gas regions having temperatures higher than $8000 \mathrm{~K}$ as in the stellar chromosphere or chromospherelike regions (Giampapa et al. 1978; Howell et al. 2006b). In addition, the strengthening of the chromospheric activity line indicators is consistent with stellar activity phenomena which, depending on the secondary-star dynamo magnetic field, might trigger episodes of enhanced mass transfer rate as observed in our epoch 2 "bursting" spectra.

Acknowledgements. E.M. thanks Vincenzo Andretta for his help providing valuable inputs about chromosphere activity. E.M. also thanks the ESO director general for having supported a science leave in Bologna where the paper was concluded and the Bologna IASF/INAF institute for the kind hospitality. The authors wish also to thank the anonymous referee for the precious suggestions and comments while reviewing the first draft.

\section{References}

Andretta, V., Doyle, J. G., \& Byrne, P. B. 1997, A\&A, 322, 266 Araujo-Betancor, S., Gänsicke, B. T., Hagen, H.-J., Rodriguez-Gil, P., \& Engels, D. 2003, A\&A, 406, 213

Araujo-Betancor, S., Gänsicke, B. T., Long, K. S., et al. 2005, ApJ, 622, 589

Barman, T. S., Hauschildt, P. H., \& Allard, F. 2004, ApJ, 614, 348

Cowley, A. P., Crampton, D., \& Hutchings, J. B. 1982, 259, 730

Diaz, M. P., \& Steiner, J. E. 1994, A\&A, 283, 508

Gäensicke B. T., Long K. S., Barstow M. A., \& Hubeny I. 2006, ApJ, 639, 1039

Giampapa, M., Schneeberger, T. J., Linsley, J. L., \& Worden, S. P. 1978, ApJ, 226,144

Horne K., \& Schneider D. P. 1989, ApJ, 343, 888

Howell, S. B., Ciardi, D. R., Dhillon, V. S., \& Skidmore, W. 2000, ApJ, 530, 904 Howell, S. B., Harrison, T. E., Campbell, R. K., Cordova, F. A., \& Szkody P. 2006a, AJ, 131, 2216

Howell, S. B., Walter, F., Harrison, T. E., et al. 2006b, ApJ, 652, 709

Kafka, S., Honeycutt, R. K., \& Howell, S. B. 2006, AJ, 131, 2673

Kafka, S., Howell, S. B., Honeycutt, R. K., \& Robertson, J. W. 2007, AJ, 133, 1645

Mason, E., Wickramasinghe, D., Howell, S. B., \& Szkody, P. 2007, A\&A, 467, 277

Mukai, K. 1988, MNRAS, 232, 175

Pandel D., \& Cordova, F. A. 2005, ApJ, 620, 416

Rottler, L., Batalha, C., Young, A., \& Vogt, S. 2002, A\&A, 392, 535

Schmidt, G. D., Smith, P. S., \& Harvey, D. A. 1995, AJ, 110, 398

Schmidt, S. J., Cruz, K. L., Bongiorno, B. J., Liebert, J., \& Reid, I. N. 2007, AJ, 133,2258

Schneider, D. P., \& Young, P. 1980, ApJ, 240, 871

Schwope, A. D., Mantel, K.-H., \& Horne, K, 1997, A\&A, 319, 894

Schwope, A. D., Staude, A., Koester, D., \& Vogel, J. 2007, A\&A, 469, 1027

Thoroughgood, T. D., Dhillon, V. S., Steeghs, D., et al. 2005, MNRAS, 357, 881

Steeghs, D., Marsh, T., Knigge, C., et al. 2001, ApJ, 562, L145

Uchida Y., \& Sakurai T. 1985, in Unstable Current Systems and Plasma Instabilities in Astrophysics, ed. M. R. Kundu \& G. D. Holman (Dordrecht: Reidel), IAU Symp., 107, 281

Warner, B. 1995, Cataclysmic Variables Stars (Cambridge University Press)

Young A., Skumanich A., \& Paylor V. 1988, ApJ, 334, 397 